\title{
Os sete aspectos considerados nas tarefas de passagem da Aritmética para a Álgebra
}

The seven aspects considered in the tasks of transition from Arithmetic to Algebra

Lourival Pereira Martins ${ }^{1}$ Marlene Alves Dias ${ }^{2}$

\section{Resumo}

Neste artigo são apresentados os sete aspectos considerados como importantes para o ensino e aprendizagem da Álgebra, quando se pondera sobre a passagem da Aritmética para a Álgebra. Iniciamos pela exposição das definições e exemplos dos sete aspectos considerados, os quais são associados às pesquisas de Chevallard, Robinet e Duval, que auxiliaram na identificação e definição desses aspectos. A metodologia utilizada na pesquisa consistiu em: análise documental, diagnóstico de estudantes de uma escola privada desde o Ensino Fundamental ao Ensino Médio e avaliações de um dos autores enquanto professor de estudantes do Ensino Superior. Na exposição dos sete aspectos, apresentamos alguns exemplos encontrados no decorrer da pesquisa. Concluímos que é preciso desenvolver esses aspectos durante a Educação Básica para que os estudantes possam aplicá-los em diferentes tarefas, em particular, naquelas que correspondem ao seu cotidiano e às suas aspirações profissionais.

Palavras chave: aspectos da álgebra; passagem da aritmética para a álgebra; educação básica.

\section{Abstract}

In this article we present the seven aspects considered important for the teaching and learning of algebra when considering the transition from Arithmetic to Algebra. We begin by exposing the definitions and examples of the seven aspects considered, which are associated with the researches of Chevallard, Robinet and Duval, that helped in the identification and definition of these aspects. The methodology used in the research was the documentary analysis, the diagnosis of students from a private elementary school to high school and assessments as a teacher of higher education students. In the discussion of the seven aspects we present some examples found during the research. We conclude that it is necessary to develop these aspects during the basic education so that the students can apply them in different tasks, in particular, those that correspond to their daily life and their professional aspirations.

Keywords: aspects of algebra; transition from arithmetic to algebra; basic education. 


\section{Introdução}

Iniciamos a pesquisa sobre os diferentes aspectos associados à álgebra a partir dos estudos realizados por Chevallard (1984, 1989, 1990) e Robinet (1989). Estes estudos nos permitiram identificar e definir sete formas de interagir quando se consideram tarefas que envolvem a álgebra escolar. Estas sete formas de tratamento das tarefas escolares configuram-se em diferentes aspectos da álgebra e foram identificadas por: memória, linguagem, generalização, equivalência da igualdade, equivalência entre ostensivos, estrutura e análise, uma vez que esses aspectos correspondem às habilidades cognitivas a serem aprendidas pelos estudantes durante seu desenvolvimento mental.

Nas pesquisas envolvendo o ensino e aprendizagem da Álgebra, detectamos que uma das dificuldades talvez esteja relacionada à falta de compreensão de que, ao se trabalhar com essa área do conhecimento matemático/humano, é necessário que o sujeito tenha domínio de determinadas habilidades, podendo utilizá-las de forma consciente, articulandoas na solução das tarefas associadas à Álgebra que the são propostas durante toda a Educação Básica.

A escolha do termo aspecto está associada ao seu sentido na língua portuguesa, uma vez que ele se origina do latim aspectus, que significa a forma de ver, observar um dado objeto, no caso um objeto matemático e é com esta acepção que empregamos esse vocábulo neste estudo. Consideramos que o referido termo não está associado apenas ao modo de ver esse objeto, mas também ao papel por ele desempenhado na realização de tarefas envolvendo a Álgebra escolar, isto é, à maneira como é utilizado na solução das tarefas propostas aos estudantes.

Apresentamos a seguir os sete aspectos por meio de definição e exemplos que podem facilitar sua compreensão.

\section{Os sete aspectos da álgebra identificados na pesquisa}

- O aspecto de memória: no cumprimento de tarefas que, segundo Chevallard (1998), corresponde a qualquer atividade humana que o indivíduo se propõe a realizar; em particular, nos interessam as tarefas matemáticas. No caso dessas tarefas, é comum o encadeamento de uma sequência de subtarefas, para as quais é necessário que quem a realiza não perca de vista a tarefa principal. É preciso desenvolver a tarefa mantendo a memória dos elementos utilizados para resolvê-la de forma que estes indiquem o caminho seguido e possibilitem o controle e a sustentação do questionamento inicial. Como exemplo desse aspecto, consideramos a utilização do algoritmo na divisão de dois números naturais ou decimais utilizando o que se convenciona chamar de método da chave. Neste caso, as operações sucessivas, ao serem anotadas, viabilizam um controle sobre o desenvolvimento das mesmas e facilitam a execução da tarefa principal.

Chevallard (1984) considera que uma das etapas fundamentais na construção da Álgebra está relacionada exatamente ao desenvolvimento de um sistema de anotações que funcione como uma memória para manter o controle das operações efetuadas. Dessa forma, quando falamos de aspecto de memória, não estamos nos referindo à habilidade de lembrar um determinado conhecimento, mas de uma estratégia de anotação (registro) que possibilite ao sujeito resgatar uma informação durante a execução da tarefa. 
Um exemplo que nos permite visualizar esse aspecto pode ser observado na divisão de polinômios. Consideremos a divisão do polinômio $2 x^{3}-8 x^{2}-18 x+12$ pelo polinômio $x^{2}-6 x$ +3 , cuja solução, usando o método da chave, se assemelha muito - para não dizer que é igual - ao método relacionado a esse tipo de divisão, quando se consideram dois números, ou seja, em ambos os casos podemos aplicar o algoritmo de Euclides.

Voltando à divisão dos dois polinômios indicados acima pelo método das chaves, que se representa da seguinte forma $2 x^{3}-8 x^{2}-18 x+12 \mid \underline{x^{2}-6 x+3}$, iniciamos dividindo 0 termo de maior grau do dividendo pelo termo de maior grau do divisor e multiplicando o resultado obtido pelo divisor entre eles, a saber: 2x. Desse modo, considerando a importância do registro das operações efetuadas, deve-se indicar o resultado da multiplicação de $2 x$ por $x^{2}-6 x+3$, cujo produto será subtraído do dividendo como indicado na sequência.

$$
\begin{aligned}
& 2 x^{3}-8 x^{2}-18 x+12 \mid \frac{x^{2}-6 x+3}{2 x} \\
& -\underline{2 x^{3}+12 x^{2}-6 x} \quad 2 \mathrm{x}
\end{aligned}
$$

O resultado da subtração, sendo $4 x^{2}-24 x+12$, é registrado no desenvolvimento do método.

$$
\begin{aligned}
& 2 x^{3}-8 x^{2}-18 x+12 \mid \frac{x^{2}-6 x+3}{2} \\
& \frac{-2 x^{3}+12 x^{2}-6 x}{0+4 \mathrm{x}^{2}-24 x+12} \quad 2 \mathrm{x}
\end{aligned}
$$

Observamos que o novo polinômio encontrado, a saber: $4 x^{2}-24 x+12$, tem o mesmo grau que o polinômio do divisor, logo, efetuamos agora a divisão entre o monômio $4 x^{2}$ e o monômio $x^{2}$, cujo resultado é igual a 4 . Na sequência, multiplicamos 4 pelo polinômio divisor e subtraímos o resultado como registrado no desenvolvimento do método, obtendo resto igual a zero.

$$
\begin{gathered}
2 x^{3}-8 x^{2}-18 x+12 \mid \frac{x^{2}-6 x+3}{2 x+4} \\
\frac{-2 x+12 x^{2}-6 x}{0+4 \mathrm{x}^{2}-24 x+12} \\
\frac{-4 \mathrm{x}^{2}+24 x-12}{0}
\end{gathered}
$$

Ou seja, $\left(2 x^{3}-8 x^{2}-18 x+12\right) \div\left(x^{2}-6 x+3\right)=2 x+4$ e tem resto zero, ou ainda, $2 x^{3}-$ $8 x^{2}-18 x+12=\left(x^{2}-6 x+3\right) \cdot(2 x+4)+0$. Nesse algoritmo, observamos que sucessivas anotações levam a um controle da realização da divisão desejada, sendo possível resgatar as informações referentes à mesma sem maiores dificuldades. Ressaltamos ainda que ao considerar o resultado sobre a forma $2 x^{3}-8 x^{2}-18 x+12=\left(x^{2}-6 x+3\right) \cdot(2 x+4)+0$ corresponde a outro ostensivo para representar o não ostensivo algoritmo de Euclides que é também uma maneira de controlar o resultado encontrado.

- O aspecto de linguagem: a comunicação do saber ganhou grande impulso com a invenção da escrita e, segundo Duval (1995), "não é possível estudar os fenômenos relativos aos conhecimentos sem recorrer à representação". No campo da Matemática, podemos 
considerar que o desenvolvimento de um sistema que permitisse não apenas representar como também comunicar esse saber foi um dos aspectos que representou uma das maiores contribuições para o avanço, tanto dessa área como de todo o conhecimento humano. Exemplo disso pode ser observado na formulação de um sistema de representação numérica eficiente, o sistema de numeração decimal, que, como é possível verificar na gênese do cálculo algébrico, foi fundamental para a construção dessa nova linguagem.

De acordo com Chevallard (1984), a representação na linguagem algébrica, como um instrumento de descrição de problemas numéricos, é uma ferramenta avançada, pois proporciona não só a comunicação dos resultados, mas também a sua generalização. "A linguagem algébrica - notadamente por ser uma memória - propicia conservar da melhor maneira a informação apresentada e, sobretudo, indicar a informação pertinente" (CHEVALLARD, 1984, p.75, tradução nossa).

Por exemplo, ao desenvolver expressões como 25 - 2. $(7+3)$, a representação da informação pode ser feita registrando o resultado à direita, ou na linha seguinte da expressão, ou seja, $25-2 .(7+3)=25-2$. 10, mas para isso ser possível, foi necessária a criação de um sinal - o de igualdade - que permitisse a ligação entre as duas informações equivalentes. Nesse exemplo, observamos que o que estamos considerando como aspecto de linguagem não se refere apenas à linguagem algébrica, mas a uma gama de formas de registro semióticos que levam à execução das tarefas e à comunicação de seus resultados. De forma mais específica, nessa gama de linguagens, temos a linguagem coloquial, falada e escrita, usada na comunicação de forma geral, como destacado por Duval (2011). Desse modo, avaliamos o aspecto de linguagem como aquele que possibilita comunicar a informação de forma precisa sem dubiedades.

- O aspecto de generalização: de acordo com Chevallard (1984), a introdução da Álgebra no ensino na França é historicamente marcada pelo que se pode considerar uma dialética entre o velho ensino da Aritmética e o novo ensino da Álgebra. Segundo o autor, antes da introdução da chamada Matemática Moderna, a introdução do novo conhecimento se dava a partir de problemas da Aritmética, para os quais era proposta a solução Aritmética, seguida de uma solução Algébrica e da apresentação dos sinais usuais que o autor denomina sinais aritméticos, ou seja,,,$+- x_{1}=$, etc.

De acordo com Robinet (1989), a introdução de letras para nomear indeterminações atende diretamente o problema prático da Matemática e faz avançar a teoria das equações, especialmente quando se refere à determinação das raízes de uma função associada às equações dadas pelos seus coeficientes, pois estas permitem a obtenção de funções que modelam as soluções por equações.

A autora indica, ao dividir as funções do cálculo algébrico segundo duas finalidades, aquela em que este pode ser considerado apenas como um meio para resolver problemas práticos, o que ela denomina Aritmética da incógnita ou a outra que o generaliza em classes de problemas que obedecem a uma mesma estrutura, o que facilita a resolução de problemas complexos.

No extrato que segue, a autora esclarece o que ela denomina duas funções da Álgebra, que indicam duas diferentes formas de tratamento do cálculo algébrico, sobre as quais deve haver uma reflexão, quando do seu ensino. 
Para os didatas que procuram problemas que são a origem de diversos conceitos matemáticos, existe sobre isso a reflexão, pois parece que o cálculo algébrico pode ser dividido em duas partes, respondendo a duas diferentes funções:

- A Aritmética da incógnita permitindo matematizar e em seguida resolver alguns problemas práticos (Aritmética em particular),

- O cálculo literal permitindo escrever as relações e tratar todos os problemas práticos originários da mesma estrutura e dando acesso a essa estrutura e, consequentemente, à resolução de problemas complexos da mesma maneira que a problemas mais simples que têm origem nessa mesma estrutura. (ROBINET, 1989, p. 12 tradução nossa).

Ao enfatizar o aspecto de generalização, observamos que é ele que torna possível a representação numérica de forma ampla, não se referindo a um valor específico, mas a uma classe de valores, cujas tarefas podem ser realizadas da mesma maneira sem que se perca a validade do resultado encontrado. É o que se observa na tradução de problemas como "A idade de Pedro é o dobro da idade de seu sobrinho Juca. Se a soma de suas idades é 39 anos, qual a idade de Pedro e de Juca?". Trata-se aqui de uma tarefa que pode ser resolvida por meio da Aritmética da incógnita, mas que, ao ser desenvolvida por via do cálculo literal, auxilia na compreensão de tarefas mais complexas.

A tarefa enunciada acima foi utilizada em nossa pesquisa diagnóstica para compreender qual a relação pessoal que os estudantes da Educação Básica mantinham com este aspecto. Observamos aqui que, consoante Chevallard (2015), a noção de relação pessoal a um objeto, para uma determinada pessoa $x$ e para um dado objeto 0 , designa 0 sistema mais ou menos integrado, mais ou menos rico, de todas as maneiras que $x$ pode se conectar com 0 .

Sendo assim, a relação pessoal esperada dos estudantes ao traduzir o enunciado era: escrever matematicamente a frase dada em língua natural, isto é, utilizar o cálculo literal para resolver a tarefa proposta: "A idade de Pedro é o dobro da idade de seu sobrinho". Consequentemente, esperava-se que após considerar a idade de Pedro como $x$, os estudantes representassem a idade de Juca, sobrinho de Pedro, como $2 x$ e a soma das idades de Juca e Pedro, como $2 x+x$, para igualá-las a 39. Neste caso, após realizar a conversão do enunciado em língua natural para a linguagem algébrica, ou seja, considerar a expressão $2 x+x=$ ?, já se supõe uma generalização para todas as possíveis situações com as mesmas características, não importando a soma das idades. Desse modo, esse aspecto cria as condições para a solução de uma classe de problemas e não apenas de problemas isolados. Segundo Chevallard (1984), é ele que amplia as potencialidades na resolução de tarefas relacionadas ao numérico.

- O aspecto de equivalência entre ostensivos: os conceitos de ostensivos e não ostensivos foram definidos por Chevallard (1994). De acordo om o autor o autor, os objetos matemáticos não são objetos comuns como uma pedra ou caneta, mas frutos da engenhosidade humana, em essência das ideias expressas sob a forma de conceitos ou definições que evoluíram ao longo de nossa história e cujo acesso se dá por meio das representações criadas ao longo de sua construção. O autor destaca que, para se compreender as técnicas que conduzem à realização dos diferentes tipos de tarefas, temos 
a necessidade de estabelecer a distinção entre dois objetos fundamentais: os objetos ostensivos e os objetos não ostensivos.

Por objeto ostensivo, o autor considera aqueles que assumem uma forma material qualquer como uma régua, a caneta, do mesmo modo que podemos considerar como um objeto material: palavras, ações, diagramas, desenhos etc. Por outro lado, os objetos não ostensivos são os normalmente chamados de noções, conceitos, ideias, entre outros. De maneira geral, os objetos não ostensivos não podem ser manipulados diretamente, pois sua manipulação se dá por meio da manipulação dos ostensivos a eles associados, como por exemplo, o conceito de número. O conceito é complexo e normalmente é confundido com os ostensivos que os representam, pois só temos acesso a eles por meio dos ostensivos que Ihes são associados.

Ainda segundo Chevallard (1994), a aplicação de qualquer técnica envolve a ativação de um complexo de objetos, alguns ostensivos, que serão manipulados, outros não ostensivos, que serão evocados. A manipulação dos ostensivos é feita por meio da evocação de determinados não ostensivos, e estes últimos, ao contrário, são evocados por meio da manipulação dos ostensivos, existindo assim uma dialética necessária entre ostensivos e não ostensivos.

Por exemplo, o conceito número dois é um objeto não ostensivo que só pode ser manipulado por meio dos ostensivos a ele associados, como o símbolo 2, a palavra dois, o sucessor natural de um, a terça parte de 6, entre outros. Assim, as tarefas formuladas para a atividade matemática dependem em grande medida da habilidade de manipulação dos ostensivos associados aos conceitos nelas envolvidos, ou seja, os não ostensivos. Assim, ao mesmo tempo em que a manipulação dos ostensivos depende da compreensão dos não ostensivos a eles associados, a compreensão dos não ostensivos depende da manipulação dos ostensivos, o que configura a dialética entre os ostensivos e os não ostensivos.

A importância dos ostensivos e não ostensivos em toda atividade matemática nos conduziu a ponderar sobre o aspecto de equivalência entre ostensivos e considerá-los como um instrumento importante que nos "mostra" como se configura o objeto matemático, ou seja, o não ostensivo, sob suas diversas formas de representação, ou ainda, por meio dos ostensivos que permitem manipulá-lo. Por exemplo, o numeral dois mil quinhentos e quarenta e três pode ser escrito no sistema de numeração decimal na forma 2.543 que é equivalente, no mesmo sistema decimal a $2 \times 1.000+5 \times 100+4 \times 10+3$, que é equivalente a $2 \times 10^{3}+5 \times 10^{2}+4 \times 10^{1}+3 \times 10^{0}$. Essas diferentes maneiras de tratar $\mathrm{o}$ numeral considerado dependem das necessidades quando da realização de diferentes tipos de tarefas.

- O aspecto de equivalência da igualdade: a Aritmética pode ser considerada uma das ferramentas mais antigas do conhecimento matemático. Sua construção está provavelmente relacionada à necessidade de controle e computação associada às nossas obrigações primárias. Esse aspecto computacional induz à obtenção de uma resposta ligada aos dados computados, o que para a grande maioria dos profissionais é suficiente.

De forma prática, levando os aspectos culturais de escrita e leitura, temos que $2 \times 3=$ 6. Em princípio, "os dois lados da igualdade ou membros" não são expressões idênticas formal ou sintaticamente -, o que já impediria escrever $6=2 \times 3$ ". (CHEVALLARD, 1984, p. 66 tradução nossa), pois na expressão numérica $2 \times 3=6$, os termos (6) à direita e $(2 \times 3)$ à esquerda do sinal de igual não são idênticos, uma vez que em Matemática um objeto só é idêntico a ele mesmo, mas esses dois termos representam o mesmo número, sendo 
portanto equivalentes, pois obedecem a determinadas propriedades, isto é, a relação de igualdade é uma relação de equivalência.

A criação de uma representação para a igualdade parece estar apenas associada às necessidades da Matemática, relacionadas ao desenvolvimento da linguagem matemática e não a uma necessidade da escrita natural. Dessa forma, o aspecto de equivalência da igualdade é aquele que torna possível reescrever, em sequência, um objeto matemático de modo a que os novos ostensivos correspondam ao mesmo não ostensivo que the é associado.

- O aspecto de análise: ao se deparar com uma nova tarefa ou situação em que as técnicas conhecidas não possibilitam uma resolução imediata, o esperado é que o indivíduo a explore na busca entre as técnicas que domina e na criação de uma nova técnica que possibilite a realização da tarefa proposta. Exemplo desse tipo de aspecto pode ser observado em tarefas como a apresentada na figura 1, que segue.

\section{Que algarismos estão faltando nesta conta?}

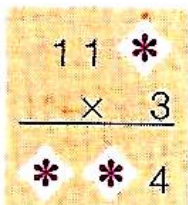

Descobrindo os algarismos e somando-os, obtemos:
a) 10
b) 12
c) 14
$x$ d) 16

Figura 1. Tarefa que utiliza o aspecto de análise (IEZZI et al, 2009, p. 57)

Nesta tarefa, é solicitado ao estudante descobrir quais algarismos estão faltando e, embora se trate de um tipo de tarefa proposta a estudantes do Sexto Ano do Ensino Fundamental, espera-se que eles explorem, entre as técnicas relacionadas à multiplicação o que se supõe já terem domínio -, aquela(s) que permita(m) obter o número que multiplicado por 3 tenha como resultado 4 na casa das unidades, no caso o número 8 . Essa análise inicial the favorecerá encontrar os demais algarismos, aplicando as técnicas de multiplicação e da soma estudadas nos anos ou séries anteriores.

Podemos considerar que o aspecto de análise é o que possibilitará, não só as resoluções dos problemas antes do advento da Álgebra, como também a utilização, tanto de métodos geométricos, como aritméticos. Sendo assim, pode ser este um dos aspectos que parece ter criado as condições para a formulação dessa nova área do conhecimento humano. Consideramos como aspecto de análise aquele que exige que o estudante explore a tarefa sob diversos ângulos na procura de conhecimentos que propiciem sua realização, o que Robert (1998) considera como nível de conhecimento disponível.

- $O$ aspecto de estrutura das operações numéricas: de acordo com Chevallard (1989), igualdades como $25-2 x(7+3)=25-2 x 10$ escondem uma dificuldade recorrente relacionada à adequação ao tipo de tarefa que se pretende realizar. Entretanto, na realização de tarefas relacionadas à Álgebra, é comum a necessidade de expressão, semelhante à anterior, bem como do uso das propriedades operatórias a ela associadas. 
Na construção das teorias, tanto da Álgebra como da Aritmética, é evidente esse tipo de disposição. Por exemplo, para o conjunto dos números naturais, podemos partir de uma definição, aparentemente intuitiva, como a de adição.

Observamos que na utilização da adição, representada entre parênteses, estão associados os aspectos de generalização e de equivalência, que tornam possível ordenar as partes para construir o todo. Esses atributos que possibilitaram o desenvolvimento de uma teoria associada à Aritmética constituem o que denominamos de aspecto de estrutura das operações numéricas. Reportando-nos a Chevallard (2015), podemos identificá-lo por meio da noção de relação institucional como pertencente ao sistema de ensino, inserido na proposta curricular, que o indica para ser estudado no quinto ano do Ensino Fundamental anos iniciais, como foi possível constatar por meio da análise dos livros didáticos da Educação Básica avaliados e propostos pelo Ministério da Educação.

Dessa forma, consideramos que o aspecto de estrutura das operações numéricas está relacionado às propriedades operatórias e suas diferentes formas de representação e aos conceitos e noções a ela associados, isto é, aos ostensivos empregados e aos não ostensivos que lhe são associados.

Para compreender o papel desempenhado pelos aspectos acima definidos, consideremos o problema "Entre todos os retângulos de perímetro $20 \mathrm{~cm}$, determine o de área máxima." (IEZZI et al., 2009, p.110). De modo geral, esse é considerado um problema algébrico, mas devido ao ostensivo numérico - perímetro $20 \mathrm{~cm}$ - pode perfeitamente ser respondido aritmeticamente, pelo método das tentativas, da seguinte maneira:

Se a medida da largura é igual a $2 \mathrm{~cm}$, a altura deverá ser de $8 \mathrm{~cm}$, logo, a área será de $16 \mathrm{~cm}^{2}$;

Se a medida da largura é igual a $3 \mathrm{~cm}$, a altura deverá ser de $7 \mathrm{~cm}$, logo, a área será de $21 \mathrm{~cm}^{2}$;

Se a medida da largura é igual a $4 \mathrm{~cm}$, a altura deverá ser de $6 \mathrm{~cm}$, logo, a área será de $24 \mathrm{~cm}^{2}$;

Se a medida da largura e da altura é igual a $5 \mathrm{~cm}$, logo, a área será de $25 \mathrm{~cm}^{2}$;

Se a medida da largura é igual a $6 \mathrm{~cm}$, a altura deverá ser de $4 \mathrm{~cm}$, o que equivale ao retângulo de largura $4 \mathrm{~cm}$ e altura $6 \mathrm{~cm}$. Pode-se concluir então que o retângulo de área máxima é o quadrado, cujos lados medem $5 \mathrm{~cm}$, uma vez que voltamos aos casos anteriores.

A solução algébrica, que é a aqui esperada, deve levar o estudante a traduzir o problema da forma como foi apresentado, ou seja, supõe-se a passagem da linguagem natural para a linguagem algébrica, o que envolve os aspectos de análise, linguagem, equivalência entre ostensivos e memória, visto que o estudante:

- Primeiro: deverá ler e compreender o texto apresentado em linguagem natural e dessa maneira se relacionar de forma satisfatória com o aspecto de linguagem;

- Segundo: buscar entre os conhecimentos de que dispõe as noções de retângulo, quadrado e suas propriedades, perímetro e área de um retângulo, o que significam e como se obtém o perímetro e a área de um retângulo, aspecto de análise;

- Terceiro: representar os não ostensivos associados ao perímetro e área de um retângulo na forma algébrica, aspectos de equivalência entre ostensivos, generalizando. $\bigcirc$ que corresponde a formular os não ostensivos perímetro e área no quadro geométrico e algébrico, apresentando a figura de um retângulo e associando letras ao mesmo ou utilizando apenas o quadro algébrico, como mostramos na sequência. 
Ao utilizar o quadro algébrico, é preciso considerar as medidas da largura, altura, e considerar as noções de perímetro e área por meio de "letras", isto é, representá-las por meio de fórmulas, o que configura a utilização dos aspectos de generalização, linguagem e memória, e conduz ao sistema: $\left\{\begin{array}{l}2 l+2 h=20 \\ l \cdot h=a\end{array}\right.$

Dessa forma, voltando ao enunciado da tarefa - Entre todos os retângulos de perímetro $20 \mathrm{~cm}$, determine o de área máxima -, observamos que será necessário introduzir novos ostensivos e não ostensivos, que correspondem às necessidades do quadro algébrico e que viabilizarão a solução algébrica do problema, que só será possível se o estudante mantiver bom relacionamento com os saberes envolvidos e os aspectos da álgebra a eles associados, como:

- de equivalência da igualdade, estrutura e equivalência entre ostensivos e não ostensivos no momento em que o estudante simplifica a equação $2 l+2 h=20$ obtendo a equação $l+h=10$;

- de estrutura, linguagem e memória, no momento em que o estudante isola uma das variáveis como a altura $h$, obtendo a mesma em função da largura l, ou seja $h=10-l$;

- de estrutura, equivalência da igualdade, equivalência entre ostensivos, generalização e memória no momento em que o estudante substitui a função $h$, na equação $l . h=a$, obtendo $l$. $(10-l)=a$, ou seja $a=10 . l-l^{2}$ que equivale à função $a(l)=10 . l-l$, função do segundo grau que apresenta valor máximo 25 para $l=5$, e encontra uma resposta para a tarefa proposta.

Desse modo, ponderando sobre o que acabamos de expor, é possível observar que o estudante que não mantiver um bom relacionamento com os sete aspectos da álgebra poderá ter dificuldade na resolução desse tipo de tarefa, em particular, em seu tratamento algébrico.

Mas, como já observamos inicialmente, a mesma tarefa pode ser resolvida utilizando apenas conhecimentos aritméticos, o que parece ser interessante de ser trabalhado com os estudantes, quando se inicia a passagem da aritmética para a álgebra, pois esse caminho mostra o caráter formalizador, unificador e generalizador da álgebra, ou seja, o que Robert (1998) indica por fug.

Aqui ressaltamos ainda que a aritmética da incógnita, como explica Robinet (1989), corresponde a uma das funções da Álgebra, logo, nos parece que a pesquisadora também compactua com nossa proposta que corresponde a mostrar a possibilidade de tratamento, tanto pela aritmética como pelo cálculo literal, quando se trata da passagem da Aritmética para a Álgebra.

Salientamos ainda a importância do aspecto linguagem, pois o estudante com dificuldade no relacionamento pessoal com o aspecto de linguagem corre o risco de não compreender o enunciado do problema proposto e, neste caso, poderá ter dificuldade também na conversão entre a linguagem natural e a linguagem algébrica. Nesse caso, parece interessante a proposta de Duval (2011), que indica a importância de se proporem exemplos simples de conversão entre a linguagem natural e a linguagem algébrica, de modo a auxiliar o estudante que poderá desenvolver, compreender o problema por meio de uma solução aritmética, que pode se realizar por intermédio de uma tabela de valores que permita, no final, trocar valores particulares por letras e assim justificar o caráter fug da Álgebra e mostrar a economia de utilizá-la, quando da resolução de determinadas tarefas. 
O estudante com dificuldade no relacionamento pessoal com o aspecto de análise poderá considerar difícil identificar os não ostensivos associados às definições e propriedades das figuras envolvidas no problema, o que impedirá a realização da tarefa. Aqui, observamos que a articulação entre os quadros geométrico e algébrico é uma ferramenta importante para o desenvolvimento da Álgebra, mas é preciso que os estudantes mobilizem os conhecimentos geométricos propostos nas tarefas de introdução à Álgebra.

O estudante com dificuldade no relacionamento pessoal com o aspecto de memória pode não conseguir organizar as informações obtidas na interpretação do problema proposto. Trata-se aqui de uma habilidade específica que precisa ser considerada, em particular, quando se propõem tarefas que ultrapassam a simples reprodução de técnicas desenvolvidas em aula. Para o caso do exemplo em que é preciso articular quadro geométrico e algébrico, a disposição dos elementos em uma tabela pode ser interessante para se refletir sobre a solução aritmética e fazer a passagem para a solução algébrica.

O estudante com dificuldade no relacionamento pessoal com os aspectos de equivalência da igualdade, equivalência entre ostensivos e generalização, corre o risco de não conseguir representar algebricamente as informações obtidas a partir do problema inicialmente proposto, o que reforça a importância de mostrar o caráter fug (formalizador, unificador e generalizador) por meio da passagem da Aritmética para a Álgebra, ou seja, mesmo que a tarefa possa ser resolvida facilmente por meio da Aritmética, é importante compreendê-la algebricamente para poder aplicar os conhecimentos desenvolvidos em tarefas mais complexas.

O estudante com dificuldade no relacionamento pessoal com o aspecto estrutura poderá ter problema quando do desenvolvimento do sistema de equações, o que impossibilita a obtenção da função quadrática que permite encontrar a solução da tarefa.

Ressaltamos que os sete aspectos considerados precisam ser introduzidos continuamente no processo de ensino e aprendizagem de forma a auxiliar os estudantes a construírem um conjunto de conhecimentos que lhes possibilite tratar tarefas que ultrapassem a aplicação de definições e técnicas, que os habilitem na aplicação desses conhecimentos em tarefas contextualizadas, em particular, aquelas que encontramos no cotidiano ou nas diferentes profissões.

Um exemplo das dificuldades que podem ser geradas em função da falta de relação pessoal com os sete aspectos considerados na pesquisa é a tarefa exposta em Martins et Dias (2013) ao analisar a solução proposta por um estudante do Ensino Superior para a questão:

O salário de um representante comercial de filtro de água é composto por um valor fixo de $R \$ 1.100,00$, acrescido de $R \$ 9,00$ a cada filtro vendido. Determine:

A expressão que permite obter o salário do vendedor em função da quantidade de filtros vendidos.

Quantos filtros terão sido vendidos se o salário bruto desse vendedor for de $R \$$ $2.180,00 ?$

Na figura 2, a seguir, apresentamos um extrato da solução proposta por um estudante de um curso superior em uma das avaliações feitas pelo professor. 


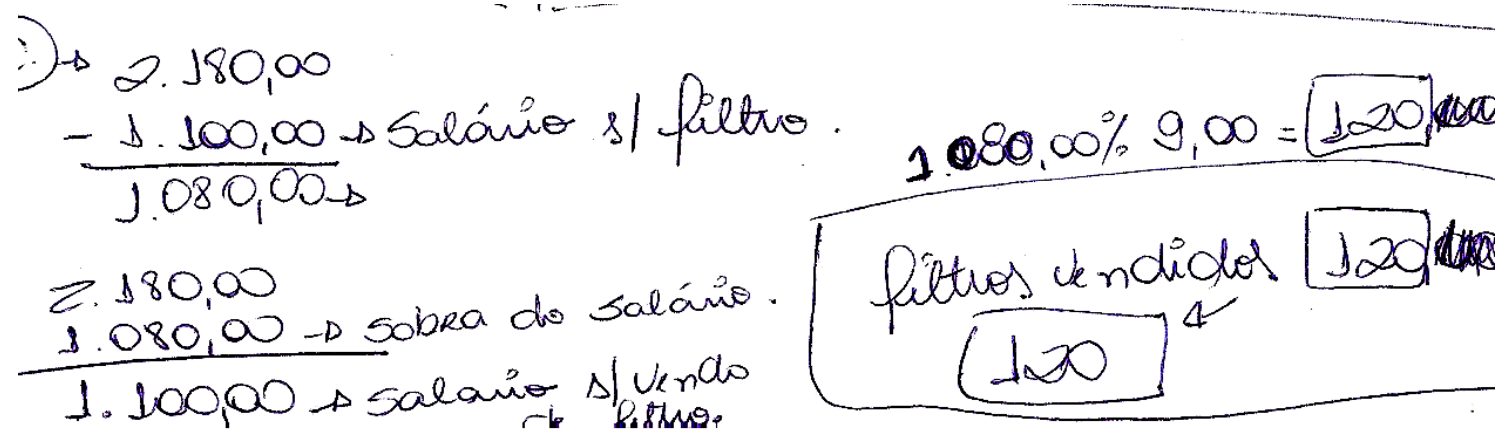

Figura 2. Solução proposta por um estudante do Ensino Superior em uma avaliação de Matemática (MARTINS, 2015, p. 42)

O estudante não apresentou a resposta para o item a, que tinha por objetivo levá-lo a converter o ostensivo apresentado em linguagem natural para os ostensivos na linguagem algébrica, ou seja, considerar o aspecto de equivalência entre ostensivos. Entretanto isso não o impediu de responder ao item b, para o qual era possível utilizar apenas o quadro da Aritmética, o que, em geral, ocorre com tarefas desse tipo.

Analisando a solução apresentada, observamos que o estudante demonstra ter um bom relacionamento com os aspectos de linguagem, visto que conseguiu compreender a tarefa e responder $\mathrm{o}$ item $b$, utilizando os aspectos de análise, pois mobilizou os conhecimentos necessários para a solução do problema e de memória, uma vez que registrou de forma clara o raciocínio empregado, utilizando os ostensivos numéricos e língua natural, possibilitando assim um desenvolvimento de forma lógica e coerente da tarefa proposta.

A solução apresentada é essencialmente aritmética e a não realização do item a pode indicar dificuldades por parte do estudante no relacionamento com os aspectos de generalização, equivalência entre ostensivos, equivalência da igualdade e estrutura, o que dificulta a realização de tarefas mais complexas relacionadas à Álgebra por parte desse estudante. Sendo assim, deve-se estar atento ao fato de que é preciso propor tarefas em que a Álgebra seja necessária e ultrapasse a simples aplicação em tarefas escolares que não têm sentido, quando se trata de aplicar os saberes desenvolvidos na escola em situações profissionais e/ou cotidianas.

Outro exemplo que acreditamos ser importante destacar foi o apresentado por um estudante do Ensino Médio na solução de dois problemas propostos na atividade diagnóstica relacionada à pesquisa. No extrato da figura 3, podemos visualizar a solução do referido estudante. 


\section{5) Resolva os problemas abaixo}

a) Quando Ana nasceu seu irmão tinha sete anos. Se a soma das idades dos dois é 35 anos, qual a idade de Ana? $35-f=28$

$$
\text { R: a volode de Ar a } 2 \text { and }
$$

b) A idade de Pedro é o dobro da idade de seu sobrinho Juca. Se a soma das idades dos dois é 39 anos, qual a idade de Pedro e Juca?

$$
\begin{aligned}
& 2 x+1 x=39 \\
& \frac{39}{3}=x=13
\end{aligned}
$$

Figura 3. Extrato de solução apresentada por um estudante do Ensino Médio (MARTINS, 2015).

Observamos que para a segunda tarefa, o estudante utilizou uma solução algébrica, revelando manter uma relação pessoal pelo menos parcial com os sete aspectos da Álgebra. O estudante demonstra bom relacionamento com o aspecto de equivalência da igualdade, visto que apesar de não indicar uma passagem intermediária, 3x = 39, consegue manipular essa equivalência, o que pode ser observado pela escrita, $\frac{39}{3}=x=13$, mesmo com uma falta de organização, o que pode indicar uma dificuldade em relação ao aspecto de linguagem e memória.

Esse indicativo parece ficar evidente nas anotações relativas à primeira tarefa, com o estudante não obtendo sucesso na realização da mesma. As anotações indicam que ele se limitou a efetuar a diferença entre trinta e cinco e sete, concluindo que a idade de Ana é de vinte e oito, como fica claro na resposta apresentada.

Isso nos leva a levantar as seguintes hipóteses:

- O estudante não compreendeu o enunciado, interpretando que Ana tinha sete anos a mais que seu irmão, o que evidentemente não está de acordo com o enunciado, sinalizando dificuldade com o aspecto de linguagem e análise.

- O estudante compreendeu o enunciado e buscou a solução aritmética, efetuando a diferença entre trinta e cinco e sete. Entretanto, parece ter ocorrido uma perda de conexão entre o momento em que leu e interpretou o enunciado e o momento em que estava realizando a tarefa, ou seja, apresentou dificuldade no relacionamento pessoal com o aspecto de memória, linguagem e equivalência entre ostensivos. De qualquer forma, podemos considerar que o objeto inicial se perdeu durante a realização da tarefa, com o estudante não lembrando que trinta e cinco era a soma das idades dos dois irmãos e Ana tinha sete anos a menos que seu irmão.

Bosch e Chevallard (1999) destacam o papel dos ostensivos discursivos na realização de tarefas como a apresentada acima. Embora o estudante não tenha finalizado a resolução da tarefa de forma satisfatória, a solução parcial apresentada se aproxima da forma dos raciocínios envolvidos numa solução aritmética da seguinte forma:

Se Ana tem 7 anos a mais que seu irmão e a soma de suas idades é 35 anos, então se retiramos 7 de 35 equiparamos as duas idades, ou seja, Ana e seu irmão teriam juntos 28 anos se tivessem a mesma idade, logo teriam 14 anos cada. Como Ana tem sete anos a mais que seu irmão então terá 14 + 7, ou seja, Ana tem 21 anos e seu irmão 14 anos. 
Chevallard (1990) destaca que problemas deste tipo, bem como o utilizado no primeiro exemplo envolvendo a função quadrática, dificultam o trabalho com a Álgebra, pois podem ser resolvidos aritmeticamente e o estudante não vê motivos para apreender uma nova ferramenta para resolver situações que ele conseguiria sem a mesma. O pesquisador argumenta ainda que a solução aritmética para esses tipos de problema é muito mais econômica que a algébrica, o que dificulta ainda mais o despertar do interesse por parte dos estudantes para o estudo dessa área do conhecimento humano.

\section{Considerações finais}

Acreditamos que estamos longe de identificar todos os fatores que dificultam o estabelecimento de uma relação pessoal eficiente com os objetos da Álgebra e dessa forma encontrar meios que conduzam ao uso dos saberes relacionados a esse ramo do conhecimento humano que, dependendo de nossas escolhas, pode estar cada vez mais presente em nosso cotidiano. Por exemplo, para os desenvolvedores de softwares, os objetos da álgebra são essenciais, pois se consideramos o momento em que o funcionário da companhia de água vem medir nosso consumo, podemos verificar que existe um software remoto que efetua os cálculos para diversas quantidades de consumo, ou seja, nesse software foi programada uma função por intervalos que viabiliza o cálculo.

Entretanto observamos, por meio da pesquisa, que é necessário desenvolver uma relação pessoal com os sete aspectos da álgebra, definidos para as análises, se desejamos que os estudantes construam, no decorrer da Educação Básica, um conjunto de competências e habilidades que os auxiliem a tratar diferentes tipos de tarefas, dispondo de conhecimentos matemáticos que possam ser aplicados em tarefas do cotidiano e de suas escolhas profissionais.

Exemplos simples que indicam essa necessidade são o da interpretação de gráficos que indicam a variação do dólar e o da leitura e interpretação do consumo mensal de água ou luz que são apresentados de forma bastante simples com necessidade de utilização apenas do quadro aritmético, mas que podem ser melhor compreendidos quando se dispõe de conhecimentos associados ao quadro algébrico.

\section{Referências}

BOSCH, M. et CHEVALLARD, Y. La sensibilité de l'activité mathématique aux ostensifs. França, 1999. Disponível em: <http://yves.chevallard.free.fr/spip/spip/IMG/pdf/Sensibilite_ aux_ostensifs.pdf>. Acesso em: 17 jun. 2017.

CHEVALLARD, Y. Pour une aproche anthropologique du rapport au savoir. Dialogue 155, Paris, jan. 2015. Disponível em: <http://www.gfen.asso.fr/images/documents/publications/ dialogue/dial155_enligne_anthropo_rap_savoir_chevallard.pdf>. Acesso em: 16 jun. 2017.

CHEVALLARD, Y. Organisations didactiques: 1. les cadres généraux. França, 1998. Disponível em: <http://yves.chevallard.free.fr/spip/spip/article.php3?id_article=38>. Acesso em: 17 jun. 2017. 
CHEVALLARD, Y. Ostensifs et non-ostensifs dans I'activité mathématique. França, 1994. Disponível em: <http://yves.chevallard.free.fr/spip/spip/article.php3?id_article=125>. Acesso em: 17 jun. 2017.

CHEVALLARD, Y. Le passage de l'arithmétique a l'algébrique dans des mathématiques au college. Troisième partie. França, 1990. França. Disponível em: <http://www-irem.ujfgrenoble.fr/revues/revue_x/fic/23/23x1.pdf>. Acesso em: 17 jun. 2017.

CHEVALLARD, Y. Le passage de l'arithmétique a l'algébrique dans l'enseignement des mathématiques au college. Deuxième partie. França, 1989. Disponível em: <http://wwwirem.ujf-grenoble.fr/revues/revue_x/fic/19/19x5.pdf>. Acesso em: 17 jun. 2017.

CHEVALLARD, Y. Le passage de l'arithmétique a l'algébrique dans l'enseignement des mathématiques au college. Première partie. França, 1984. Disponível em: <http://wwwirem.ujf-grenoble.fr/revues/revue_x/fic/5/5x3.pdf>. Acesso em: 17 jun. 2017.

DUVAL, R. Sémiosis et pensée humaine. $1^{a}$ ed. Paris: Peter Lang, 1995.

DUVAL, R. Ver e Ensinar a Matemática de outra Forma - Entrar no modo Matemático de pensar: os registros de representações Semióticas. 1ª ed. São Paulo: Editora PROEM, 2011.

IEZZI, G. et al. Matemática - Ciências e Aplicações. Vol. 1 São Paulo: Editora Atual. 2009.

MARTINS, L. P.; DIAS, M. A. Diagnóstico de alguns aspectos que dificultam a passagem da Aritmética para a Álgebra na Educação Básica no Brasil. Curitiba, 2013. Disponivel em: <http://sbem.esquiro.kinghost.net/anais/XIENEM/comunicacoes_6.html>. Acesso em: 17 jun. 2017.

MARTINS, L. P. Estudo sobre aspectos da Álgebra na passagem da Aritmética para a Álgebra. Tese de Doutorado. Universidade Anhanguera de São Paulo. São Paulo. SP. 323 p. 2015.

ROBERT, A. Outils d'analyse des contenus mathématiques à enseigner au lycée à l'université. Recherches en didactique des Mathématiques, vol. 18, n. 2, p. 139-190, 1998.

ROBINET, J. La genèse du calcul algébrique (une esquisse). França, 1989. Disponivel em: <http://www.irem.univ-paris-diderot.fr/up/publications/IPS97065.pdf>. Acesso em: 17 jun. 2017. 